\title{
CHALLENGES THAT THE REFERRAL SYSTEM POSES TO CARE GIVING AS EXEMPLIFIED BY THE KANYE COMMUNITY HOME- BASED CARE (CHBC) PROGRAMME IN BOTSWANA
}

\section{Simon Kang'ethe}

\section{OPERATIONAL DEFINITION}

While a caregiver is generally a person who volunteers to take care or help in caring for sick persons generally at home, whether family members, relatives or other community members, the study distinguishes between the primary caregiver who usually stays with the clients at all times, and the community caregiver (so referred to as a volunteer in the Botswana context) who is a government-recruited community volunteer and moves from house to house to help the primary caregivers execute their caring roles.

The four concepts: sick person, client, patient and HIV/AIDS client/s are used interchangeably.

\section{STUDY RATIONALE}

While the broad study on which this article is based aimed at assessing the contribution of caregivers to Community Home-Based Care programmes, taking Kanye CHBC programme as a platform, the aim of this article is to explore and discuss the challenges and bottlenecks that the current referral system faces, as well as the effectiveness of care giving in Kanye and Botswana generally.

\section{BACKGROUND AND INTRODUCTION}

A referral system is a network providing services to the clients from one level of service delivery to another, depending on the needs of the client. In Botswana, the referral network is based on a structure comprised of successive levels of services and activities, with each one having its own level of service capacity and sophistication. The ladder of service delivery from the lowest level of sophistication starts with the health mobile points, health posts, clinics, primary hospitals, district hospitals and the national referral hospitals (MOH, 1991). However, following the operational treatment hierarchy may not be applicable in some settings, as some levels of the hierarchy may not be present at some settings. In Gaborone, for example, patients are referred from the clinics to Princess Marina, a national referral hospital, and therefore bypass both the primary and district-level hospitals. The purpose of the referral system is to ensure equitable distribution of effective health care and equitable health resources to all Botswana. However, it should be noted that services at lower levels of the referral ladder, that is mobile points, are minimal and ineffective, with available human resources usually being family welfare educators whose capacity to diagnose and prescribe is limited. It is therefore important that other levels are well equipped and satisfactory in service delivery. The absence of this would mean people becoming dissatisfied and losing confidence and interest in taking their clients to the referred facility. However, the referral system in Botswana is well grounded and informed by policies and guidelines, with the provision that clients in the referral points are well attended and monitored (MOH, 1991).

The country of Botswana is one of the countries in the developing world with adequate health facilities. It is estimated that $85 \%$ of the population is within a $15 \mathrm{~km}$ radius and $76 \%$ within walking distance of such facilities (MOH/NACP 41, 1998). According to referral policy guidelines, health providers refer their patients needing additional care to the next level of the 
system; provide adequate written information such as consent forms describing the patient's condition and the reasons for referral; and arrange as much as practical support, e.g. transport, for the patient and, if possible, have the client accompanied to the referral point. In the context of $\mathrm{CHBC}$, before a client is referred for home-based care, the policy guidelines states that a hospital referral discharge letter has to be released to the caregiver of the client/patient $(\mathrm{MOH}$, 1991; NACP 30, 1996).

Cross-referral, especially between the clinics and private practitioners, is also important as some clinics lack some medical items, while others are well stocked. In the context of CHBC service provision, a short supply of, for example gloves in one clinic may be corrected by patient referral to another clinic for the service. It is worth noting that any referral case from the private practitioners to the government health facility is treated the same as any other case within the government system. However, the referral for corrective services such as counselling, either provided by non governmental organizations (NGOs), community based organizations (CBOs) or private individuals, complements this and makes service delivery efficient (MOH, 1991; NACP 30, 31, 1996).

The efficiency and existence of the referral network determines to a greater extent the quality of care that a client gets in home-based care. This is because most community home-based care clients get sick sporadically, meaning that prompt and fast attention, coupled with the availability of the needed services and medication, are central to effective treatment and their convalescence. Availability of essential care package and medication in the clinics or referral points and the prompt availability of transport are central to the success of the programme (Kang'ethe, 2004, 2006; NACP 13, 1993).

\section{STUDY SETTINGS AND METHODS}

\section{RESEARCH DESIGN}

A qualitative design was used in the study. This is because the study sought to explore the thinking, feelings and attitudes of the caregivers towards the challenges that the current referral system presents to care giving.

\section{SELECTION CRITERIA AND PROCEDURE}

All the 140 registered primary caregivers were picked for inclusion in the study, with 82 (59\%) registered primary caregivers presenting themselves for ten focus group discussions. The focus group discussions were intended to collect qualitative data on the views, attitudes and thinking of the caregivers pertaining to the contributions of caregivers and to explore the challenges and gaps presented by other factors such as the state of the referral system. All the four CHBC nurses and their coordinator were also selected to participate in the study. The primary caregiver, therefore, formed the unit of analysis (Merriam, 1988; Neuman, 1997). The caregivers' selection criteria and grouping disregarded age and gender, and were based on the caregivers' clinic/health post proximity and where one was being served, with some clinics having more than one focus group discussions. The caregiver interviewees were selected from the following health facilities: Kgwatlheng, Kanye main, Mafhikana, Dada, Mmamokhasi clinics and Dilolwe and Sebogo health posts. The discussants were collected from their clinics and taken to the Miracle Family Christian Centre premises in Kanye, where focus group discussions were conducted. However, the CHBC nurses were each interviewed at their place of work. 


\section{RESEARCH INSTRUMENTS}

An interview guide was used to steer 10 focus group discussions with respondents in each discussion group varying from six to twelve. The instrument had been tested and refined during the pilot study. Discussions were tape recorded. With only slight changes, the same interview guide was used to administer one-to-one individual interviews with the nurses who formed the caregivers' supervision team. The role of the CHBC nurses' responses was to corroborate, cross-check and confirm the responses and information from the caregivers.

\section{DATA ANALYSIS, INTERPRETATION AND BIAS REDUCTION}

To carry out data analysis, both sets of information from the focus group discussions with primary care givers and the one-to-one interviews with the nurses were taped and then transcribed. The huge mountain of primary or crude data was reduced to manageable categories and themes that formed the basis of analysis. Quotes, analogies and proverbs were used, while tables and graphs were used to present the data and infer the findings. To reduce data bias and ensure data reliability and validity, results from the pilot study findings acted as baseline data. There was double translation of the instruments, that is translation from English to Setswana and then from Setswana to English by two independent translators, the two translators coming together to settle on the difference. However, to strengthen reliability and validation of the data, the four nurses and their coordinator answered more or less the same questions with the primary caregivers. The two interview guides or the instruments differed only slightly and the two sets of responses were confirmed, corroborated and cross-checked against each other.

\section{ETHICAL AND LEGAL REQUIREMENTS}

To ground the study in a legal and ethical environment, all appropriate ethical issues were taken into consideration: informed consent, maintenance of confidentiality and anonymity, adequate debriefing and consultation before commencement of the study, no coercion, and treating the respondents with all due respect to maintain their integrity and their human rights (Creswell, 1994; Neuman, 1997). The researcher had complied with all the research permit application procedures from the department of the Research and Development Committee Board (HRDC) and therefore given a research permit. The researcher then wrote to the Southern District Council asking for authority to enter into the field to collect data. The Southern District Council (SDC) matron then wrote letters to all the Kanye heads of clinics requesting them to cooperate and help the researcher to pursue the data-collection exercise.

\section{RESEARCH DOMAIN}

The data for this article were obtained from empirical research done in December 2005 and January 2006 at Kanye village, which has a population of 40,628 persons (CSO, 2001). The village is well endowed with adequate health facilities in that it has a big Seventh Day Adventist (SDA) referral hospital, five clinics and two health posts. In the Botswana context, a clinic is bigger in terms of the extent of human resources and facilities, and it serves a bigger population than a health post would. This research domain was chosen as the area was experiencing a high HIV prevalence rate of 25,8\% and was experiencing a progressively higher death toll among the CHBC clients (NACA, 2005). The researcher also felt that a deeper research analysis of the conditions surrounding care giving was necessary, as the HIV/AIDS dynamics continuously presented new challenges. It was also the interest of the researcher to document his experience towards writing a PhD Dissertation as he had worked for five years in the area. 


\section{FINDINGS/RESULTS}

\section{Profile of the volunteer caregivers}

\section{Age, gender and educational dimensions of the caregivers}

The study findings indicated that the caregiver participants' ages ranged from 18 to 85 years, with 46 caregivers (56\%) being 50 years and above; 28 of them being $60(34 \%)$ years and above. The study revealed that most caregivers were women and, especially in the case of those above 60 years, were apparently financially handicapped and physically not strong enough to meet the care-giving demands. Seventy-two (88\%) caregivers had no income to support themselves. Their poverty was demonstrated by some caregivers breaking into tears as they explained the economic and social environments in which they carried out their care. Because of the poor referral system and shortage of information, many were apparently aware of only limited sources of help.

The relatively elderly caregivers especially indicated they were psychologically drained by care giving and that they needed to be assisted, especially by the youth, and also by the men. Many indicated not knowing other avenues of help, like where they could get help such as counselling other than in the clinics. This was an indicator of gaps in the referral system.

Findings on literacy indicated that $74 \%$ of the caregivers had either never been to school or had only primary-level education, with only $5 \%$ of the caregivers having tertiary education. Illiteracy was found to contribute to low care productivity and poverty. This was psychologically disenabling as most of the illiterates, who were also elderly, had problems of accessing education on care giving; following the medical and hygiene protocol; and following the progression of the disease of their clients (Atta \& Fidzani, 1996; Kang'ethe, 2006). On findings relating to gender, the study findings indicated that the Kanye programme faced a seriously gender-skewed dimension, with 80 (98\%) being women and two (2\%) being men. Since in many societies of the world women have other domestic chores, care giving presented a condition of being overburdened and societal exploitation. This, according to the feminists, contributes to the subjection of women to poverty and presents a human rights abuse dimension (Finch, 1984; Kelesetse, 1998; UNDP, 1995; Walker, 1982).

\section{INADEQUATE CARE PACKAGE, MEDICAL FACILITIES AND REFERRAL SYSTEM}

Seventy-two (88\%) of the Kanye research participants confirmed that the referral system was weakened by inadequate medical and care package facilities. They stated that the care package offered by the clinics to the caregivers was inadequate as it does not contain all the necessary tools needed for care giving. The caregivers indicated that the situation never used to be like that a few years back and that the decline in the care package could be associated with poor health facilities and CHBC programme management. In some wards important tools such as gloves, napkins, cotton wool and bandages are not included in the care package and the supply is irregular and unreliable (Kadzakumanja, 2001; Kang'ethe, 2004). The four CHBC nurses confirmed that most of the tools needed for care giving were not available. Because of inadequate napkins, for example, there was a directive from the clinic administration that they only be given to clients experiencing incontinence. This caused a rift and conflict between the caregivers and the nurses, with caregivers seeing the process as discriminatory and unfortunate. Though there was a provision that one could get what is not available from another clinic, the networking system or referral between the clinics was not well operationalised and was failing. 
The following quotes testify to the insufficiency of the care package:

"These days there is nothing in the clinics. The caregivers are suffering”.

"The necessities to help the sick are no longer there. We are not helped well”.

“We are all going to die from contagion. We do not know our status".

"The care package components are not adequate. Sometimes there is nothing in the clinics".

"The other clinics do not easily give you what your clinic does not have, even though you have been directed by the nurse".

Since the care package forms the basic support structure for the caregivers, inadequate provision therefore implies that the care-giving process suffers, with the resultant compromise on the quality of care.

\section{Inadequate knowledge on disease progression and referral system}

The majority of the Kanye research participants confirmed that the state of client referral was aggravated by the fact that most of the caregivers were illiterate and were not in a good position to adequately track down progression of the disease. This is supported by the fact that $74 \%$ of the Kanye caregivers had either never been to school or had only primary-level education, with only $5 \%$ of the caregivers having tertiary education. The elderly caregivers indicated that, though it was possible to know when their client was improving, they were not adequately tracking a decline in their client's condition. The research findings also indicated that the Kanye programme had no training plan for caregivers. This is indicated by the fact that caregivers had not been exposed to any form of training for years. The training gap is glaring and detrimental to providing quality care in the programme.

\section{Heavy caseload and poor working conditions at Kanye SDA hospital influence referral}

Almost all the research respondents expressed sadness about the conditions prevalent at the SDA referral hospital in Kanye village. They indicated that the condition is deplorable and that the sick are not given adequate attention. The doctors are rarely seen and it was trainees who dealt with the clients most of the time. The hospital did not have workers either to wash the sick or to feed them. It is the caregivers and their close relatives who are expected to wash and feed their clients. They further noted that the hospital was overwhelmed with sick people, too many for the staff to handle. This was negatively affecting the quality and effectiveness of the referral system. Caregivers made the following comment to express their disappointment:

"We are like 'doctors' in the hospital, though without the right tools for work".

"Some appointments with doctors fail because of their heavy caseload".

These quotations indicate their frustration, as caregivers come on all the appointed days to wash and clean their clients, with scheduled appointments usually failing. This usually led to frustration and possible psychological breakdown. Caregivers, therefore, preferred the clients to be handled within the home environment, where the caregiver can get a little respite and avoid hospital visitation and frustration every day. The respondents also reported that most of their clients preferred to be taken care of at home (NACP 31, 1996; Sims \& Moss, 1991). 


\section{Inadequate assistance from health care providers}

According to the majority of the respondents, the poor state of referral was attributed to inadequate psychological and emotional support from the health care providers. The four CHBC nurses agreed with the caregivers that the health team only did a minimal job in the care-giving process, as it was only in a few cases that they visited the caregivers and their clients. This normally happened with new clients who needed to be referred. However, such cases were usually rare. The health care providers' visit schedule was exacerbated by transport problems. The respondents reported not having access to counselling services for psychological support. This, according to the caregivers, left them not knowing when making progress or failing. Some said that some clients were dying because appropriate advice or counselling was not given to the caregivers in good time. The caregivers commented the following:

"We do not get counsellors to offer counselling in our caring duties".

"We do not see the health personnel coming to encourage us. We rarely get counselling".

Care giving, being psychologically draining and burdensome, calls for remedial services to caregivers to help invigorate and replenish their energy to maintain a state of well-being. Respondents, therefore, felt a dire need for counselling, debriefing, supervisory visits and incentives, among other things.

\section{Attitudes of health service providers towards clients and their caregivers}

Forty-two $(50 \%)$ caregivers stated that most of the medical providers had poor public relations skills and a negative attitude towards the caregivers and their clients. For example, they indicated that most service providers were not adequately respectful and were rude to them. Respondents further indicated that, while the health personnel have gloves and masks for protection, these are not made available to the caregivers and their relatives who come to attend to their clients. The hospital authorities sometimes demanded that caregivers leave the hospital before finishing attending to their clients. This treatment made the clients and their caregivers reluctant to use the services rendered, which was affecting the referral process. These caregivers indicated that it was for that reason that some caregivers and their clients were looking for alternative treatment from traditional healers and opting for being attended to at home (NACP 31, 1996; UNAIDS, 2000a).

\section{Stigma negatively influences referral}

Sixty (73\%) caregivers reported that even though the stigma was declining with time in Kanye and in Botswana generally, it was still affecting the treatment and therefore referral of clients to points of assistance. The caregivers revealed that some caregivers and family members were hiding their clients and therefore shutting the avenues of help for the clients. Caregivers reported having knowledge that some people were taking their clients to places further away such as the towns of Lobatse, Jwaneng or even Gaborone city. This is because of the stigma attached. However, since taking the clients to farther places was expensive, sometimes it was impossible, and this in turn usually affected the referral and treatment efficiency of such clients.

\section{Transport challenge}

Eighty (98\%) caregivers admitted that the challenge of finding transport was seriously affecting the referral system. Though they admitted that the council had a vehicle that was supposed to help in the referral, it was not well managed and organised. They reported that in most of the cases when they telephoned the council officers for vehicle assistance, they hardly got assistance in time, or there was no assistance at all. This meant that caregivers were stressed to 
a point of burnout as they struggle to get assistance, sometimes totally failing to take their clients in time. Sometimes they would fail to make referral and appointments dates, which was to the detriment of the health of the client.

\section{Management of the CHBC programme}

Forty primary caregivers (49\%) caregivers indicated that the CHBC programme was not well managed. First they indicated that they were discriminated against in favour of the community caregivers (volunteers). For any knowledge package in the form of workshops, it was the community caregivers who were prioritised. They were also the ones who were given things like T-shirts, yet they felt that they were the ones who were on 24-hour duty for the clients. They also indicated that even the decline in the care package was a result of poor management. They noted that sometimes the clients are released to community home-based care programmes prematurely. This they said they understood was because of congestion in the referral hospital.

\section{DISCUSSION OF THE FINDINGS}

\section{Demographics}

The Kanye study findings confirmed that most caregivers are elderly women. Other studies in Botswana had found more or less the same condition. Atta and Fidzani (1996) found that over $50 \%$ of caregivers in most of the Botswana CHBC programmes are elderly women who may not adequately stand up to care-giving demands. This definitely impacts negatively on the quality of care giving. Leaving care giving to such elderly women is tantamount to threatening the traditional safety nets providing for care giving (McDonnel, Brennan, Burnham \& Tarantola, 1994). Such elderly persons may not adequately understand the dynamics of care giving and seek referrals in time. Periodic training of these caregivers on care giving, especially on available avenues of referrals is critical (Abbat \& Meija, 1988; Petit, 1994). The government and care managers need to increase community education and advocacy to attract especially the younger and the educated to share in the caring duties. Young and knowledgeable people are also in a position to understand the HIV/AIDS disease well and other avenues of assistance for the client referral.

As in the Kanye study, many caregivers of HIV/AIDS clients the world over are women (UNAIDS, 2001b). A study carried out in Botswana by Munodawafa (MOH/NACP 41, 1998) had all females as respondents, except one in Tutume, while in Molepolole, all caregivers were females. In this era of HIV/AIDS it is not fair to leave the caring tasks to women alone. They need to be assisted by their male counterparts. Due to the dynamics of HIV/AIDS, it is becoming very difficult to cope. According to Kelesetse (1998), a feminist, CHBC has shifted state responsibilities to women. This is therefore a case of gender exploitation and presents a human rights violation. This also contributes immensely to the feminisation of poverty (UNDP, 1995). Gender mainstreaming and socialisation intervention strategies should be carried out in all the institutions of the country, especially the schools. This will ensure that children embrace gender neutral norms, values and thinking from childhood. The boys as well as girls should grow up knowing that care-giving tasks are to be undertaken by all. Advocacy education by leaders and civil society bodies is necessary to show that care giving should be a task for all in society (MLHA, 1999; UNDP, 1995).

On the findings of the low educational status of many caregivers in Kanye, other studies in Botswana by Phorano, Nthumang and Ngwenya (2005) in Maun and Kweneng found that 33\% of the caregivers studied had only lower primary education. To offset the impact of such low 
literacy levels, there should be an emphasis in the programme on adult informal education and on-the-job training by care managers, in collaboration with the government, to ensure that there is adequate knowledge and that the coping capacities of the caregivers are improved (Abbat \& Meija, 1988; Petit, 1994). As in the Kanye study, age has always been found to be a factor that negatively affects the quality of care giving. A study by Jacques and Stegling (2001) in Kweneng, Botswana, found three caregivers who were too old to adequately discharge their roles. Studies by Erikson (1959), and Wilkinson and Campbell (1997) indicate that the change towards senility is marked by a decline in cognitive, emotional and physical energies, affecting one's productivity holistically. This leaves doubt as to the capacity of these elderly caregivers in understanding referral and care giving generally. Research conducted in Thailand, Zimbabwe and Uganda found that over two thirds of elderly respondents were the main caregivers of ill adults or orphans whose parents died of HIV/AIDS, while further extensive research done in Thailand found that most of the caregivers taking care of HIV/AIDS sufferers were 50 years and over, with $40 \%$ being at least 60 years (Ntozi \& Nakayiwa, 1999; UNAIDS, 2001a). This unexpected burden in their later years is a heavy one, particularly if they have no previous knowledge of HIV/AIDS or of basic home-care techniques such as universal precautions. On-the-job training and education in the avenues of help for referral are critical (Abbat \& Meija, 1988). Socialising the young from childhood by inculcating non-gender-based values and education through educational institutions could ensure that boys as well as girls take care giving as the job for all.

The phenomenon of inadequate medical facilities is common in many resource-strained countries, especially in the developing world (WHO, 2002). Health care providers are frequently confronted with clinical challenges like infections and tumours that call for prompt and appropriate treatment. Treatment of these infections and tumours is of great importance, as it decreases suffering and prolongs life, especially in the absence of effective and non-toxic antiretroviral therapy against HIV (NACP 13, 1993). In the context of CHBC, the inadequate care package has resulted in conflicts between caregivers and their clients. In a study by Dant and Gully (1994), though conducted in a Western setting, clients demanding what was not available caused conflict between the clients and their caregivers. Inadequate medical supplies also caused some caregivers and their clients to look for alternative medication from traditional healers. However, use of both medication from modern medication and traditional medication has always presented a conflict, with the client likely to have his or her health decline (Kang'ethe, 2004, 2006; UNAIDS, 2000a). Research in Kanye by Kang'ethe (2004) indicates caregivers becoming frustrated because of the unreliability of the care package supplies, while another study by Kadzakumanja (2001) in Lilongwe, Malawi indicates that in clinics the care package supplies are irregular, late or not available at all. This seriously affects the referral process.

It is possible that widening the radius and scope of the referral system between the clinics and other private practitioners could possibly mitigate the challenge. This is because in his field research the researcher noted that, while some clinics lacked so many of the care packages as recommended by the government (NACP 30, 1996), some clinics had a substantial supply. While some caregivers would have to be buying gloves and incontinence sheets, other clinics may have them in stock. Therefore inter-clinic referral and communication should be strengthened to improve service delivery. The government needs to honour its policy obligation of providing especially the full care package as spelt out in NACP 30 policy document (NACP 30, 1996). 
Various writers attribute the inadequate knowledge on disease progression to the fact that some caregivers are too old and have an inadequate educational background. In research by Mojapelo, Ditirafalo, Tau and Doehlie (2001) in Botswana, four clients did not have caregivers, and three clients were taken care of by very old caregivers who could not guarantee effective service delivery, while studies by Atta and Fidzani (1996) found that over 50\% of caregivers in most of the Botswana CHBC programmes are elderly women who may not be able to meet care-giving demands adequately. According to Kang'ethe (2006), inadequate knowledge stems from lack of training. Lack of training is an indicator of the following:

- Likely low skills in care giving;

- Likely low capacity to handle prevention;

- Likely low coping skills and more prone to stress and burn out;

- Likely poor hygiene and poor medical protocol;

- Likely low capacity to handle conflicts with the client.

These factors are disenabling and likely to adversely affect the capacity of the caregivers' decision to refer the client to medical facilities.

In the same study by Atta and Fidzani (1996) it was found that only $10 \%$ of the caregivers had been exposed to training. Coupled with illiteracy and lack of training, $11 \%$ of the caregivers they studied found the instructions on the administration of the medicine too complicated and would not know the appropriate time to refer the clients to help systems or to take the clients to the referral hospital. Therefore the time to take the clients to the referral hospital would sometimes be delayed to the detriment of the client's health. Petit (1994) emphasises the need and importance of periodical training to the service provider (caregiver), if good care results are to be realised. The government should ensure through care managers that all caregivers are exposed to periodic training so that they can understand the progression of the disease of their clients and therefore afford them prompt and effective referral.

Poor conditions at hospitals occur in many countries and continents, especially in poor and resource-strapped countries (WHO, 2002). A study by Atta and Fidzani (1996) found the following flaws and shortcomings in the Botswana referral system: (a) improper counselling of the patients; (b) non-counselling of the patients; and (c) lack of communication between the hospitals and the clinics which receive the patients. Further literature in Botswana indicates that referral hospitals are usually overburdened by clients, especially in this era of HIV/AIDS (MOH, 1991; NACP 30, 31, 1996). This has made service delivery slow and sometimes led to neglect of the clients. The 1996 statistics from the two national referral hospitals of Nyangabgwe in Francistown and Princess Marina in Gaborone show that patients with HIV and related diseases occupied over $50 \%$ of hospital beds in the medical and paediatric wards, while information in 2002 revealed that the bed occupancy rate for most general medical wards was over $100 \%$, while the occupancy rate for medical wards in general referral hospitals was over 133\%. While the Average Length of Stay (ALOS) for all patients was five days, those with HIV/AIDS, was 9 to 14 days (NACA, 2002:ii; NACP 38, 1997). Since the biggest hurdle in the Kanye referral hospitals and others is inadequate staffing, the government needs to employ more doctors and nurses and improve remuneration to the already employed staff to reduce staff turnover.

Inadequate assistance from the health providers generally affects the service provision and referral process in many health care settings. Across the board in many care programmes in 
Botswana the referral system is faced with the challenge of inadequate health care staff (Nurses Association of Botswana, 2004). Several studies in Botswana indicate that many caregivers and their clients, for instance, lack qualified counsellors to provide the needed psychosocial support to enable them to handle the challenges inherent in the HIV/AIDS situation successfully and be able to effect referral in time adequately (Jacques \& Stegling, 2001; Kang'ethe, 2006; Mojapelo et al., 2001). Generally across districts, the Department of Social and Community Development (S\&CD) is especially understaffed, meaning that the services of the social workers to counsel and assess the economic circumstances of the HIV/AIDS clients and their caregivers for possible government assistance and referral hardly works well. This has implications of HIV/AIDS clients getting sick and sometimes dying while waiting for the government food basket, and possible referral advice (DMSAC Report, 2005; Kang'ethe, 2004, 2006).

Clear treatment guidelines are also lacking in many care settings of the world, especially in the developing world. Many health care workers have often not received training in the management of HIV/AIDS (Abbat \& Meija, 1988; NACP 13, 1993; Nowlen, 1988; Petit, 1994; UNAIDS, 2001b), thus complicating and compounding the challenge of referral. In Botswana this negates the principles and aspiration of Botswana's vision 2016 of a safe, healthy and secure nation (Vision 2016, 1997). In a study by Atta and Fidzani (1996) in Botswana, 94,5\% of the caregivers they studied indicated lack of support from health workers, and 57,8\% of patients reported not having been visited by any health worker for a long time. Caregivers preferred health workers to visit the households in order to supervise them, to teach them and to encourage the patients to take their drugs. While the family welfare educators in Botswana are supposed to be at homes advising and directing the activities of the caregivers, Joy Phumaphi, former Minister of Health, observed that they remained in the clinics (Botswana Daily News, 2002) leaving the caregivers and their clients with inadequate support.

Subjective information in Kanye village of client neglect or clients being left in the hands of children is not uncommon (Kang'ethe, 2004). In a study by Mojapelo et al. (2001), it was found that two clients were taken care of by children. In such circumstances client disease progression and their referral cannot be guaranteed. The programme should ensure that all the clients are taken care of and their health and social needs addressed. In absence of caregivers, the programme should have a provision to allow such clients to go to hospitals and stay there. Though this would defeat the purpose of the CHBC programme, it would be ethically right to do so, if being at home means jeopardising the life of the client. The government needs to ensure that service delivery has enough drivers. The health staff should also adhere to their professional code of ethics enshrined in their job description, which requires them to deal with their tasks seriously and professionally (MOH, 1991).

The literature attributes negative attitudes by the health care providers towards clients and caregivers to their inadequate skills and exposure, and the heavy caseloads that sometimes make them suffer from burnout (Bennet, Ross \& Kelaher 1993; Dant \& Gully, 1994; MOH/NACP 41, 1998; NACP 31, 1996). Subjective information in the Kanye programme suggests that the community caregivers (volunteers) sometimes opt to go to the houses of the apparently well-to-do clients/primary caregivers, leaving behind those clients who were in abject poverty. There were also accusing fingers pointed at some of the nurses who broke the clients' confidentiality irresponsibly by disclosing the clients' status to the community. This created fear, inadequate confidence in the health facilities and affected the referral system and spirit. Health service providers should undergo a paradigm shift to treat all the clients equally 
irrespective of their economic position. They should also stick to their professional code of ethics and the policy guidelines they accepted on their employment to serve all without discrimination and know that it is legally and ethically wrong to disclose the client's status $(\mathrm{MOH}, 1991)$. Training the community caregivers in service professionalism and ethics could help change their mindset.

Stigma remains a major obstacle in the HIV/AIDS campaign generally in many countries of the world (UNAIDS, 2000b, 2001). Some caregivers in the Kanye programme could not reveal their clients' status to help systems and therefore effect their referral and possible assistance as a result of the prevalent stigma, in Thailand neighbourhood communities were accused of stigmatising HIV/AIDS clients (UNAIDS, 2001a). Festus Mogae, President of Botswana, stated that the stigma and discrimination surrounding the HIV/AIDS disease remain one of the greatest barriers to the implementation of various care and prevention strategies (Botswana Millennium Development Goals Status Report, 2004), while Kalanke (2004), states that people living with HIV/AIDS (PLWHAs) resort to using and adopting alternative coping strategies such as avoidance, denial and secrecy because of the fear associated with stigma and discrimination of HIV/AIDS. This has had serious repercussions in negatively affecting the rate of consumption of services meant to help patients and to cushion the effect of the virus on those who are affected. It also affects the health of the clients and also the referral system, as clients fail to go to the health facility as a result of denial and avoidance (Fako \& Linn, 2003).

In Kalankes' study the caregivers interviewed denied being HIV positive and thus did not utilise certain welfare services such as the food basket because of stigma attached to the programmes. This was a big drawback to care giving as most of the PLWHAs in the country happen to be poor and need help from their caregivers and other help systems. It shuts all avenues of assistance or any possible referral (Jacques \& Stegling, 2001; Kang'ethe, 2006; Khan \& Stegling, 2000; Mojapelo et al., 2001). The government, organisations in the care field and civil society organisations should increase their anti-stigma campaign to normalise responses to HIV/AIDS. This would make client referral to health facilities and other sources of help easier (Tirisanyo Catholic Commission, 1993; UNAIDS, 2000b).

Lack of transport to effect referrals in good time is usually a common problem and critically affects the quality of service delivery and the whole spectrum of the referral system $(\mathrm{MOH}$, 1991). While many CHBC programmes in Botswana are supposed to have ample transport to assist the clients to get to the hospital or the main referral hospital, the transport system is very poor and sometimes unavailable. Subjective evidence suggests that cases of clients being taken to the hospital by donkeys are not uncommon. This is usually done after efforts to contact the CHBC transport facility fails. Sometimes a vehicle could be available, but there is no driver. The CHBC programme should ensure adequate transport at all times to assist the clients in the referral. Communities should also contribute more and be ready to offer clients transport.

Inadequate care programme management also poses referral challenges in community homebased care programmes in Botswana. Under a normal and ideal CHBC situation, prior and robust arrangements need be in place before releasing the HIV/AIDS client to home-based care. For instance, it is necessary to know who will be taking care of the client, what the help system at his/her disposal is, and his/her suitability for the task. The economic and social environment of the home where the client is being discharged to needs to be assessed for possible help intervention. It is only after such an assessment by the home-based care team that a discharge sheet can be issued. However, subjective evidence of many CHBC discharge cases in Botswana 
attests to the fact that clients are discharged without any such preparatory arrangements on the ground (Jackson, 2002; NACP, 30, 31, 1996).

\section{CONCLUSION}

The referral system is dynamic and complex, its success depending on how well the officers in the whole spectrum of health facilities communicate and cooperate. Proper communication, however, requires a spirit of cooperation and a commitment to the principle of doing what is best for the patients. Inadequate referral systems or networks pose huge challenges to the health of the clients, the effectiveness of care giving and the HIV/AIDS campaign generally. It kills the motivation and interest of the caregivers in using the health facilities, making them use other alternative assistance avenues, which may be detrimental to the clients' health. The government and other non-governmental organisations in the care field need to make sure that all the health facilities are well equipped with adequate staff as well as facilities to handle the clients satisfactorily. All the health facilities need to upgrade their communication systems with one another to be effective in the referral system. The government and organisations in the care field also need to do research and identify all the factors impeding the referral system and put in place measures to address the problems. This would also make caregivers aware of all possible avenues of assistance to their clients.

\section{REFERENCES}

ABBAT, F.R. \& MEIJA, A. 1988. Continuing the education of health workers. Geneva: World Health Organisation.

ATTA, J.K. \& FIDZANI, N.H. 1996. Baseline study for Community Home Based Care Programme for Terminally ill HIV/AIDS patients in Botswana. NACP 31. AIDS STD unit, Gaborone, Botswana.

BENNET, L., ROSS, M. \& KELAHER, M. 1993. The impact of working with HIV/AIDS on health care professional: dimension and measurement. In: VANDIS, H. \& VAN DONGEN, E. (eds) Burnout in HIV/AIDS health care and support. Amsterdam University Press: 11-12.

BOTSWANA DAILY NEWS, 15th August 2002.

BOTSWANA MILLENNIUM DEVELOPMENT GOALS STATUS REPORT. 2004.

CRESWELL, J.W. 1994. Research design. Qualitative \& quantitative approaches. Thousand Oaks, Calif.: Sage Publications, Inc.

CSO. 2001. Central Statistics Office. Gaborone.

DANT, T. \& GULLY, V. 1994. Coordinating care at home. London: Collins.

DMSAC REPORT. 2005. District Multi-Sectoral AIDS Committee Report presented at Kanye Rural Administration Centre (RAC) Council Chambers. November.

ERIKSON, E.H. 1959. Identity and the life cycle. Psychological Issues, 1:1-171.

FAKO, T.T. \& LINN, G.J. 2003. Reflections on psychological, social and economic implications of HIV and AIDS in Botswana. Journal of Management Studies, 18(2).

FINCH, J. 1984. Community care: developing non-sexist alternatives. Critical Social Policy, 9:6-18.

JACKSON, H. 2002. AIDS AFRICA. Continent in crises. Safaids, Avondale, Harare, Zimbabwe. 
JACQUES, G. \& STEGLING, C. 2001. Panacea or perfidy? Paper presented at the 3rd International Conference on Social Work in Health and Mental Health, July 1-5, 2001, Tampere, Finland.

KADZAKUMANJA, O. 2001. Improving the quality of community care in Lilongwe, Malawi. Published Abstract no 103 on $1^{\text {st }}$ Regional CHBC Conference, March 2001 held at Boipuso Hall.

KALANKE, K. 2004. Influence of stigma and discrimination on coping strategies of people living with HIV/AIDS in Botswana. A case of COCEPWA and BOCAIP. Gaborone: University of Botswana. (Unpublished MA Degree in Development Studies)

KANG'ETHE, S.M. 2004. Issues and challenges of community home based care in Africa. Unpublished Masters of Social Work research essay. Gaborone: University of Botswana.

KANG'ETHE, S.M. 2006. Contribution of caregivers in community home based care programmes: the case of Kanye, Botswana. Mafikeng Campus: University of North West. (Unpublished PhD Dissertation in Social work)

KELESETSE, N.M. 1998. AIDS, home based care \& the status of women in Botswana. A case study of Mogoditshane. University of Botswana, faculty of Sociology, Gaborone: University of Botswana, Faculty of Sociology. (BA Thesis)

KHAN, B. \& STEGLING, C. 2000. An evaluation of the Kweneng district AIDS Home Based Care Programme. Gaborone: SNV, Netherlands Development Organisation.

McDONNEL, S., BRENNAN, M., BURNHAM, G. \& TARANTOLA, D. 1994. Assessing and planning home based care for persons with AIDS. Health Policy and Planning, 9(4):429-437.

MERRIAM, S.B. 1988. Case study research in education: A qualitative approach. San Francisco: Jossey-Bass.

MLHA. 1999. National Gender Programme. Women's Affairs Department. Gaborone: Government Printer.

MOH/NACP 41. 1998. Evaluation of the CHBC pilot project in Molepolole \& Tutume Sub District. NACP 41 Gaborone, February 1998.

MOH. 1991. Patient referral. Health Management System. Gaborone: Government Printers.

MOJAPELO, D., DITIRAFALO, T., TAU, M. \& DOEHLIE, E.2001. Client satisfaction and providers perspectives of Home Based care in Kweneng District, Botswana, Gaborone. (Unpublished Report)

NACA. 2002. Botswana 2002 Second Generation HIV/AIDS Surveillance. A Technical Report, November 2002.

NACA. 2005. Botswana Second Generation HIV/AIDS Surveillance. Technical Report.

NACP 30. 1996. Community home based care for people living with AIDS in Botswana. AIDS STD Unit, Gaborone.

NACP 13. 1993. Botswana guidelines for district, referral, and primary hospitals. Clinical management of HIV infection in adults. AIDS STD Unit. World Health Organisation.

NACP 31. 1996. Baseline study for the community home based care programmes for terminally ill HIV/AIDS patients in Botswana. Gaborone, Botswana. 
NACP 38. 1997. M.T.P.11, Botswana HIV and AIDS. Second medium term plan.

NEUMAN, W.L. 1997. Social research methods. Qualitative and quantitative approaches. Boston, London, Toronto, Sydney, Tokyo, Singapore: Allyn and Bacon.

NOWLEN, P.M. 1988. A new approach to continuing education for business and the professions. New York: American Council on Education \& MacMillan Publishing Company.

NTOZI, J. \& NAKAYIWA, S.1999. AIDS in Uganda: how has the household coped with the epidemic? In: ORUBULOYE, I.O., CALDWELL, J.C. \& NTOZI, J. (eds). The continuing HIV/AIDS epidemic in Africa: response and coping strategies. Health Transition Centre, Australian National University, Canberra: 155-180.

NURSES ASSOCIATION OF BOTSWANA. 2004. Caring for the caregivers. Gaborone, Kgotla Designs Pty Ltd.

PETIT, P. 1994. Closing the performance gap. Health Action, (8):4.

PHORANO, O.M., NTHOMANG, K. \& NGWENYA, B.N. 2005. HIV/AIDS home based care and waste disposal in Botswana Society. Botswana notes and records. The Botswana Society.

TIRISANYO CATHOLIC COMMISSION. 1993. Home based care. Unpublished abstract.

SIMS, R. \& MOSS, V. 1991. Terminal care for people with AIDS. London, Melbourne, Auckland: Arnold Publishers.

UNAIDS. 2000a. Collaboration with traditional healers in HIV/AIDS prevention and care in Sub-Saharan Africa. A literature review, Geneva, Switzerland.

UNAIDS. 2000b. HIV and AIDS-related stigmatisation, discrimination and denial: forms, contexts and determinants. UNAIDS Best practice Collection (UNAIDSA/00.16E).

UNAIDS. 2001a. Parents providing care to adult sons and daughters with HIV/AIDS in Thailand. UNAIDS Case Study, November.

UNAIDS. 2001b. Promoting excellence in training. The Regional Training Network (RATN). UNAIDS case study. UNAIDS Best Practice Collection. June.

UNDP. 1995. Human Development Report.

VISION 2016. 1997. Towards prosperity for all. Presidential task group for a long term vision for Botswana, September.

WALKER, A. 1982. The meaning and social division of community care. In: WALKER, A. (ed) Community care: the family, the state and social policy. Oxford: Martin Robertson: 1339.

WHO. 2002. Community home-based care in resource - limited settings. A framework for Action. 20 Avenue Appia, 1221 Geneva 27, Switzerland.

WILKINSON, J.D. \& CAMPBELL, E.A. 1997. Psychology in counselling \& therapeutic practice. Chichester: John Wiley \& Sons.

Dr Simon M Kang'ethe is a social worker for Skill Share International and attached to the Botswana Network of People Living with HIV/AIDS (BONEPWA). Botswana. 